\title{
Incidental events of diaphragmatic surgery in 82 patients with advanced ovarian, primary peritoneal and fallopian tubal cancer
}

\author{
FUMITOSHI TERAUCHI ${ }^{1}$, AIKOU OKAMOTO ${ }^{2}$, YUMIKO WADA ${ }^{1}$, \\ EI HASEGAWA ${ }^{1}$, TORU SASAKI ${ }^{1}$, OSAMU AKUTAGAWA ${ }^{1}$, \\ YASUKAZU SAGAWA ${ }^{1}$, HIROTAKA NISHI ${ }^{1}$ and KEIICHI ISAKA ${ }^{1}$ \\ ${ }^{1}$ Department of Obstetrics and Gynecology, Tokyo Medical University; \\ ${ }^{2}$ Department of Obstetrics and Gynecology, Jikei University School of Medicine, Tokyo, Japan
}

Received June 6, 2010; Accepted July 5, 2010

DOI: 10.3892/ol_00000152

\begin{abstract}
Surgical resections, such as peritoneal stripping (peritonectomy) are performed for disseminated diaphragmatic lesions of advanced ovarian cancer. This study retrospectively investigated the incidental events of diaphragmatic surgery. The records of patients with advanced mullerian carcinomas, including ovarian, primary peritoneal and fallopian carcinomas, who underwent diaphragmatic surgery were reviewed. Based on our criteria, stripping was performed for surface disease on the diaphragm, and full-thickness resection was performed for bulky disease. In certain cases, both procedures were performed. We analyzed intra- and post-operative incidental events in 82 patients. The $\chi^{2}$ and Fisher's exact tests were used in the statistical analysis. There were 82 stage III-IV cases of which 56 patients underwent stripping, 12 underwent full-thickness resection and 14 patients underwent both procedures. Unexpected open chest surgery following stripping occurred in 1 out of 63 patients $(1.6 \%)$ in the primary, 4 out of 13 patients (30.8\%) in the interval and 0 out of 6 patients $(0.0 \%)$ in the secondary debulking surgery groups. The incidence of unexpected open chest surgery was high in the interval debulking surgery group $(\mathrm{p}<0.001)$. Regarding post-operative events, accumulation of pleural effusion was identified in 43 patients $(52.4 \%)$. The incidence of pleural effusions was not significantly different between the two procedures. No complications were encountered, nor was a chest tube required, during unexpected open chest surgery and postoperative pleural effusions. Therefore, it was concluded that special attention should be paid during interval debulking surgery even though it was
\end{abstract}

Correspondence to: Dr Fumitoshi Terauchi, Department of Obstetrics and Gynecology, Tokyo Medical University, 6-7-1, Nishishinjuku, Shinjuku-ku, Tokyo 160-0023, Japan

E-mail: teralfa@tokyo-med.ac.jp

Key words: ovarian cancer, diaphragmatic surgery, debulking surgery possible for surgical resections of diaphragmatic lesions to be performed safely.

\section{Introduction}

Hoskins et al (1) reported the relationship between the diameter of residual tumors and prognosis in primary debulking surgery (PDS) in ovarian cancer. Subsequently, the importance of PDS has been examined. Although platinum preparations are usually employed, it has been concluded that tumor reduction surgery is a strong determinant factor influencing survival periods in stage III/IV patients. This conclusion was reached following a meta-analysis conducted by Bristow et al (2). The study by these authors is the only available evidence of the effectiveness of surgical therapy with respect to advanced ovarian cancer. In particular, surgical therapy was considered to be optimal when residual tumors were less than $1 \mathrm{~cm}$ since the prognosis following surgery was significantly more satisfactory, making PDS the main objective.

Patients with advanced ovarian cancer often have metastatic disease in the upper abdominal region. Nevertheless, it is difficult to achieve optimal surgery using only standard surgical techniques such as hysterectomy, oophorectomy and omentectomy, without a surgical approach that involves upper abdominal surgery (3). Using upper abdominal surgery, the prognosis after optimal surgery is significantly more satisfactory compared to suboptimal cases $(3,4,5)$. In particular, metastases to the diaphragm are common in the latter patients (6). Thus, diaphragmatic surgery is an operational procedure essential in achieving optimal debulking surgery in advanced mullerian carcinomas such as ovarian, primary peritoneal and fallopian tube carcinomas. There are two significant procedures for diaphragmatic surgery: one involves the stripping of disseminated lesions, and the other involves full-thickness resections of nodal lesions which penetrate the diaphragm. The two procedures are often performed simultaneously. Neither of these procedures is frequently performed by gynecological oncologists. In current medical practice, it appears that practitioners lacking experience in such procedures hesitate to perform them due to the possibility of incidental events. This 
Table I. Patient characteristics.

\begin{tabular}{|c|c|c|}
\hline Characteristics & No. of patients & $\%$ of 82 patients \\
\hline \multicolumn{3}{|l|}{ Age group } \\
\hline$<45$ & 18 & 22.0 \\
\hline $46-65$ & 57 & 69.5 \\
\hline$>66$ & 7 & 8.5 \\
\hline \multicolumn{3}{|l|}{ Primary cancer } \\
\hline Ovary & 71 & 86.6 \\
\hline Peritoneal & 9 & 11.0 \\
\hline Fallopian tube & 2 & 2.4 \\
\hline \multicolumn{3}{|l|}{ Histological type } \\
\hline Serous & 72 & 87.8 \\
\hline Endometrioid & 5 & 6.1 \\
\hline Mucinous & 1 & 1.2 \\
\hline Clear cell & 4 & 4.9 \\
\hline \multicolumn{3}{|l|}{ Stage } \\
\hline IIIb & 2 & 2.4 \\
\hline IIIc & 74 & 90.2 \\
\hline IV & 6 & 7.3 \\
\hline \multicolumn{3}{|l|}{ Surgical method } \\
\hline Stripping & 56 & 68.3 \\
\hline Full-thickness resection & 12 & 14.6 \\
\hline Both & 14 & 17.0 \\
\hline \multicolumn{3}{|l|}{ Surgical time } \\
\hline PDS & 63 & 76.8 \\
\hline IDS & 13 & 15.9 \\
\hline SDS & 6 & 7.3 \\
\hline
\end{tabular}

PDS, primary debulking surgery; IDS, interval debulking surgery and SDS, secondary debulking surgery. study investigated incidental events retrospectively to evaluate the safety of the two procedures.

\section{Patients and methods}

Patients. The medical records of patients with stage III-IV advanced ovarian, primary peritoneal or fallopian carcinomas were reviewed. The patients underwent diaphragmatic surgery at our institution between November 2006 and March 2009, and had preoperative and postoperative chest X-rays to detect and follow pleural effusions.

Methods. In order to obtain adequate exposure and perform diaphragmatic surgery, the infrahepatic edge of the falciform ligament containing the ligamentum teres was divided and ligated. The suture on the upper edge was left long to aid with the downward traction of the liver and remaining falciform ligament. Once the ligamentum teres was divided, the membranous portion of the falciform ligament was divided with the cautery, allowing for the liver to be separated from the anterior abdominal wall. The falciform ligament divides laterally becoming continuous with the anterior right and left coronary ligaments. The distal right hepatic vein and inferior vena cava are found beneath the dissection of the anterior right coronary ligament. For stripping, the whole area of the diaphragm (peritoneum) which contained the discoid lesions was detached or resected. On the other hand, full-thickness resections were performed only when there were obvious nodal masses penetrating the diaphragm by pre-operational imaging studies, or in cases when detachment of the peritoneum became difficult during stripping and when infiltration of deeper muscular layers was suspected. Absorbable sutures (polydioxanone: PDSII ${ }^{\circledR}$ ) were used to reconstruct the diaphragm following full-thickness resection. The chest was closed with adequate vacuuming using a suction tube which was removed immediately prior to

Table II. Clinical events among treated patients.

\begin{tabular}{|c|c|c|c|c|c|}
\hline \multirow[t]{2}{*}{ Surgical method } & \multirow[t]{2}{*}{ No. of patients } & \multicolumn{2}{|c|}{ Unexpected open chest surgery } & \multicolumn{2}{|c|}{ Pleural effusion } \\
\hline & & No. of patients & $\%$ & No. of patients & $\%$ \\
\hline Stripping & 56 & 5 & 8.9 & 25 & 44.6 \\
\hline Full-thickness resection & 12 & - & - & 7 & 58.3 \\
\hline Both & 14 & - & - & 11 & 78.6 \\
\hline
\end{tabular}

Table III. Clinical events classified by surgical time.

\begin{tabular}{|c|c|c|c|c|c|c|}
\hline \multirow[t]{2}{*}{ Clinical event } & \multicolumn{2}{|c|}{$\operatorname{PDS}(n=63)$} & \multicolumn{2}{|c|}{ IDS $(n=13)$} & \multicolumn{2}{|c|}{$\operatorname{SDS}(n=6)$} \\
\hline & No. of patients & $\%$ & No. of patients & $\%$ & No. of patients & $\%$ \\
\hline Unexpected open chest $\mathrm{t}^{\mathrm{a}}$ & 1 & 1.6 & 4 & 30.8 & 0 & 0.0 \\
\hline Pleural effusion & 33 & 52.3 & 8 & 61.5 & 2 & 33.3 \\
\hline
\end{tabular}

PDS, primary debulking surgery; IDS, interval debulking surgery and SDS, secondary debulking surgery. ${ }^{\mathrm{a} p}<0.001$. 
closing the chest. In principle, thoracic drainage tubes were not put in place.

Statistical analysis. The $\chi^{2}$ and Fisher's exact tests were used for the statistical analysis in this study.

\section{Results}

A total of 82 patients who underwent surgery at the Tokyo Medical University Hospital between 2006 and 2009 were analyzed in this study. As noted in Table I, the primary sites were the ovaries in 71 patients, the peritoneum in 9 and the fallopian tubes in 2 patients. The histological types were serous adenocarcinomas including serous papillary in 72 patients, endometrioid adenocarcinomas in 5, mucinous adenocarcinoma in 1 patient and clear cell carcinomas in 4 patients. The majority of cases were serous types.

With regard to FIGO clinical staging, 2 patients were in stage IIIb, 74 were in stage IIIc and 6 patients were in stage IV. Diaphragmatic surgery was performed during PDS in 63 patients, interval debulking surgery (IDS) in 13 and secondary debulking surgery (SDS) in 6 patients. The patients in the IDS group received 2-6 courses of paclitaxel/carboplatin combination chemotherapy prior to undergoing surgery.

Out of the 82 patients, stripping and full-thickness resections were performed in 56 and 12 patients, respectively, and the two procedures were performed in 14 patients. Diaphragmatic tumors were removed completely in 71 patients but incompletely in 11 patients $(13.4 \%)$. Regarding intra-operative incidental events, unexpected open chest surgery occurred in 5 patients $(8.9 \%)$ in the group that underwent stripping (Table II). On the other hand, post-operative incidental events including pleural effusion were observed in 43 patients (52.4\%). Pleural effusion was observed in 25 patients $(44.6 \%)$ of the group that underwent stripping, 7 (58.3\%) of the group that underwent full-thickness resection and 11 patients $(78.6 \%)$ of the group that underwent both procedures (Table II). No significant differences among the groups were noted.

In terms of the timing of the operations, unexpected open chest surgery occurred in 1 out of 63 patients (1.6\%) in the PDS group, 4 out of 13 patients (30.8\%) in the IDS group and 0 out of 6 patients $(0.0 \%)$ in the SDS group. Incidences were significantly high in the IDS group (Table III).

Of note is that none of the patients with incidental events required chest drainage or experienced problems during the natural course of the observations.

\section{Discussion}

Complete cytoreduction is the principle goal in mullerian carcinomas such as ovarian, tubal and peritoneal carcinomas. However, if this poses obstacles, efforts should be made to the greatest extent possible to make the residual disease less than $1 \mathrm{~cm}$ in order to improve prognosis (5). It has also been reported that, following the peritoneum and colon, lesions were frequently observed on the diaphragm. This hypothesis was posited by an analysis of tumor localizations in surgical reports of approximately 200 ovarian cancer cases (7). An analysis of bulky diseases in the upper abdominal region, including the diaphragm cranial to the omentum, has shown that there is a correlation between large-volume ascites and disease sites. The same study also revealed that the percentage of cases resulting in suboptimal cytoreductions increased (8). Thus, diaphragmatic disease is a crucial factor in the treatment of advanced ovarian cancer. Complete and optimal debulking rates are thought to increase if diaphragmatic lesions are removed using surgical procedures. Moreover, a better prognosis is likely to be achieved in comparison with cases which do not undergo diaphragmatic surgery $(9,10)$.

However, diaphragmatic metastases are a common obstacle to optimal cytoreduction for patients with ovarian cancer (10). Care with regard to incidental events associated with diaphragmatic surgery must therefore be exercised at all times. The most common incidental events are increased postoperative pleural effusion. It has been reported that incidences increase particularly when open chest occurs (10). With regard to postoperative incidental events, especially pleural effusion and pneumothorax, Devolder et al investigated 69 ovarian cancer patients who underwent diaphragmatic surgery (11). Of the 69 patients, 17 underwent stripping, 22 coagulation and 30 patients underwent both procedures. Postoperative pleural effusion was reported to have been observed in 41 patients, 3 of whom underwent chest drainage, 7 pleural punctures and 1 patient underwent both procedures. In addition, pneumothorax was observed in 4 out of 69 patients, and 1 of these patients required chest drainage. Moreover, Eisenhauer et al reported that ipsilateral pleural effusion was observed in more than half of the patients (an overall rate of 58\%) that underwent diaphragmatic peritonectomies or resections. These authors concluded that conservative management is possible in the majority of cases without the need for chest tubes or thorcentesis (12). Postoperative pleural effusion, which may pose clinical obstacles, was not observed in the present study of 82 patients, nor was routine chest tube placement performed at the time of stripping or full-thickness resection. Postoperative pneumothorax was observed at an overall rate of $2.4 \%(2 / 82$ patients). However, it was possible to prevent pneumothorax from occurring through the use of adequate vacuuming prior to closing the chest (13). Attention should therefore be paid when performing IDS since, as previously analyzed, the incidence of postoperative incidental events was significantly high in the case of diaphragmatic surgery following IDS. Disseminated tumors on the diaphragm, observed prior to neoadjuvant chemotherapy, are thought to induce changes such as necrosis and weakening of the layer structure of the diaphragm.

As observed in attempts at using EndoGIA stapling devices or Gore-tex grafts in diaphragmatic resections or diaphragmatic reconstructions (14), diaphragmatic surgery is likely to be actively performed in the future with respect to advanced ovarian cancer (with peritoneal or fallopian tube cancer). However, the prevention of postoperative complications and incidental events should be further investigated.

\section{References}

1. Hoskins WJ, McGuire WP, Brady MF, et al: The effect of diameter of largest residual disease on survival after primary cytoreductive surgery in patients with suboptimal residual epithelial ovarian carcinoma. Am J Obstet Gynecol 170: 974-980, 1994. 
2. Bristow RE, Tomacruz RS, Armstrong DK, et al: Survival effect of maximal cytoreductive surgery for advanced ovarian carcinoma during the platinum era: a meta-analysis. J Clin Oncol 20: 1248-1259, 2002.

3. Chi DS, Franklin CC, Levine DA, et al: Improved optimal cytoreduction rates for stages IIIc and IV epithelial ovarian, fallopian tube, and primary peritoneal cancer: a change in surgical approach. Gynecol Oncol 94: 650-654, 2004.

4. Eisenhauer EL, Abu-Rustum NR, Sonoda Y, et al: The addition of extensive upper abdominal surgery to achieve optimal cytoreduction improves survival in patients with stages IIIc-IV epithelial ovarian cancer. Gynecol Oncol 103: 1083-1090, 2006.

5. Chi DS, Eisenhauer EL, Lang J, et al: What is the goal of primary cytoreductive surgery for bulky stage IIIc epithelial ovarian carcinoma (EOC)? Gynecol Oncol 103: 559-564, 2006.

6. Kehoe SM, Eisenhauer EL and Chi DS: Upper abdominal surgical procedures; liver mobilization and diaphragm peritonectomy/resection, splenectomy, and distal pancreatectomy. Gynecol Oncol 111: S51-S55, 2008.

7. Sehouli J, Senyuva F, Fotopoulou C, et al: Intra-abdominal tumor dissemination pattern and surgical outcome in 214 patients with primary ovarian cancer. J Surg Oncol 99: 424-427, 2009.

8. Zivanovic O, Eisenhauer EL, Zhou Q, et al: The impact of bulky upper abdominal disease cephalad to the greater omentum on surgical outcome for stage IIIc epithelial ovarian, fallopian tube, and primary peritoneal cancer. Gynecol Oncol 108: 287-292, 2008 .
9. Aletti GD, Dowdy SC, Podratz KC, et al: Surgical treatment of diaphragm disease correlates with improved survival in optimally debulked advanced stage ovarian cancer. Gynecol Oncol 100: 283-287, 2006.

10. Dowdy SC, Loewen RT, Aletti G, et al: Assessment of outcomes and morbidity following diaphragmatic peritonectomy for women with ovarian carcinoma. Gynecol Oncol 109: 303-307, 2008.

11. Devolder K, Amant F, Neven P, et al: Role of diaphragmatic surgery in 69 patients with ovarian carcinoma. Int $\mathrm{J}$ Gynecol Cancer 18: 363-368, 2008.

12. Eisenhauer EL, D'Angelica MI, Abu-Rustum NR, et al: Incidence and management of pleural effusions after diaphragm peritonectomy or resection for advanced mullerian cancer. Gynecol Oncol 103: 871-877, 2006.

13. Terauchi F, Kobayashi Y, Nagashima T, et al: Pilot study on transdiaphragmatic thoracoscopic-assisted pleural biopsy and intrathoracic washing cytology for stage IIIc ovarian cancer with diaphragmatic metastases. Int J Gynecol Cancer 19: 300-303, 2009.

14. Juretzka MM, Horton FR, Abu-Rustum NR, et al: Full-thickness diaphragmatic resection for stage IV ovarian carcinoma using the EndoGIA stapling device followed by diaphragmatic reconstruction using a Gore-tex graft; a case report and review of the literature. Gynecol Oncol 100: 618-620, 2006. 